\title{
Oral Soft Tissue Precancerous and Cancerous Lesions (Excluding Salivary Glands)
}

\author{
Awni Farhan Ismael Alani* \\ Faculty of Dentistry, School of Health, BPP University, London, UK
}

*Corresponding author: Awni Farhan Ismael Alani, Faculty of Dentistry, School of Health, BPP University, London, UK, Tel: 0097339994287; E-mail: awnifarhan@yahoo.com

Received: 22 Apr, 2019 | Accepted: 10 Jun, 2019 | Published: 14 Jun, 2019

Citation: Awni F Alani (2019) Oral Soft Tissue Precancerous and Cancerous Lesions (Excluding Salivary Glands). Int J Dent Oral Health 5(4): dx.doi.org/10.16966/2378-7090.298

Copyright: (C) 2019 Awni F Alani. This is an open-access article distributed under the terms of the Creative Commons Attribution License, which permits unrestricted use, distribution, and reproduction in any medium, provided the original author and source are credited.

\section{Abstract}

The number of patients diagnosed with oral cancer is gradually increasing. According to a study report, every year around 500,000 persons are diagnosed worldwide with oral cancer. The development of precancerous lesions is an incident that involves a number of cellular, biochemical and molecular as well as clinical alterations in the fibrovascular stroma. There are several risk factors that may lead to the development of the oral precancerous and cancerous lesions. There are many investigations regarding the screening or detection methods of oral cancers. There are many options for the treatment of oral cancers which depend on various factors. Increased research in the field of oral cancer has resulted in the development of newer technologies for detection and treatment of oral cancer. With the increase in the use of tobacco and alcohol the risk of oral cancer is increasing.

Keywords: Precancerous; Cancer; Leukoplakia; Erythroplakia; Oral; Submucous; Fibrosis; Lesions; Detections; Lichen; Planus; Cancerous

\section{Introduction}

The number of patients diagnosed with oral cancer is gradually increasing. According to a study report, every year around 500,000 persons are diagnosed worldwide with oral cancer and among them around $3 \%$ are detected as malignant, which is a great threat to the world [1]. In most of the cases of oral cancer detection is delayed which increases the overall rate of morbidity and mortality. Despite of the advancement in treatment technology the overall survival rate is only $50-55 \%$ [1]. Oral cancer is very common among the older and middle aged persons i.e., after the age of 40 , but the number of young people diagnosed with oral cancer is increasing in the recent years. The studies have also shown that the risk of developing cancer is greater in case of males compared to the females. The most common oral cancer that includes almost $96 \%$ of all oral cancer cases is squamous cell carcinoma. In this assignment we are going to discuss about different types of precancerous and cancerous oral lesions, the factors that increase the risk of development of cancer along with the treatment and the new diagnostic as well as treatment advances in this field.

\section{Discussion}

\section{Clinical picture of precancerous and cancerous lesions} affecting oral soft tissues

The development of precancerous lesions is an incident that involves a number of cellular, biochemical and molecular as well as clinical alterations in the fibrovascular stroma. The clinical and pathological conditions or oral cancer include:

Leukoplakia: According to the definition proposed by the WHO, leukoplakia can be defined as a white plaque or patch which cannot be pathologically or clinically characterised as any other disease condition. Microscopically leukoplakia shows hyperplasia, acanthosis or various levels of cellular atypia and hyperparakeratosis. The most common sites where leukoplakia can be visible include alveolar mucosa, buccal mucosa and lower lip. The other probable sites of lesions include lateral tongue, floor of mouth, etc., which show malignant or dysplastic changes.

Erythroplakia: Erythroplakia is a less common oral condition, but it has been reported that it poses higher risk of progression to the malignant state compared to other precancerous oral lesions. As per the definition proposed by the WHO, it is any lesion that develops in the oral mucosa and can be seen as red, bright and velvety plaque which cannot be pathologically or clinically characterised as any other disease condition. It can be seen at any place in the oral cavity but most commonly occurs in the soft palate, mouth floor, tonsillar fauces and the ventral tongue. Generally the lesions remain asymptomatic but in some cases the patients can experience a sore or burning sensation. According to the studies the transformation rate to malignancy is from $20-68 \%$ [2].

Oral submucous fibrosis: Oral submucous fibrosis is a disease that can be described by progressive fibrosis and inflammation of the submucosal tissues i.e., deeper connective tissues and lamina propria, which leads to the development of rigidity in the mouth that in turn 
results in the failure to open the mouth [3]. The lesion may develop in any part of the mouth but the most common site that is involved in the development of the lesion is the buccal mucosa $[4,5]$. This type of lesion has a high potential to be malignant.

Oral lichen planus: Oral lichen planus is an oral mucosal disease that is characterized by its unknown etiology. It is a chronic and inflammatory disease which can be seen as white plaques or white striation or white papules. The other clinical forms of this lesion include erosion, blisters or erythema which causes pain to the patients [6]. The common sites of development of this type of lesion are the tongue, buccal mucosa, gingival etc.

\section{Risk factors for developing precancerous and cancerous lesions in the oral soft tissue}

There are several risk factors that may lead to the development of the oral precancerous and cancerous lesions. These include- Smoking increases the risk of developing oral cancerous lesions. According to a study, smokers have nine times more prone to develop oral cancer compared to non-smokers [7].

Use of marijuana may also increases the potential risk of developing oral cancerous lesions which is a common factor associated with oral cancer in youths.

The risk of development of oral cancer increases with increased use of snuff and chewing tobacco. A study carried out in women in the United States has shown that the risk of oral cancer increases by four times in persons who use snuff. Again it has been found that in case of the persons who chew tobacco, the lesions appear at the site where the tobacco is placed [8]. However, the use of smokeless tobacco poses lesser risk in developing oral cancer compared to the use of smoked tobacco, as the heat of the smoke increases the risk by many folds.

Another major risk factor is the use of alcohol which increases the risk of developing cancer in the upper aero-digestive tract. The studies have shown that moderate to heavy drinking of alcohol increases the risk of cancer by 3 to 9 times.

In Southeast Asia and India, the increased risk of oral cancer is closely associated with the excessive use of betel quid, also known as 'paan'. Chewing betel leads to the progressive development of precancerous scarring mouth condition, which is also known as oral sub mucous fibrosis.

Various studies have also detected a relation between human papilloma virus and some oral cancers. In about $22 \%$ cases of oral cancer, the cancer was detected with HPV-16 and in about $14 \%$ of cases; there is an association with HPV-18.

Dietary imbalance is also associated with elevated risk of oral cancers. Lower consumption of vegetables and fruits increases the risk.

Studies have also shown that the risk of oral cancer increases in case of patients with anaemia resulted from iron deficiency.

\section{Available investigations}

There are many investigations in the field of cancerous and precancerous oral lesions. Detection of oral cancer at the early stage is very helpful in curing the cancer. There are many investigations regarding the screening or detection methods of oral cancers.

As per the research carried out by Gillenwater A, et al. [9], auto fluorescence imaging can be used as an effective tool for the detection of oral precancerous lesions. According to them, living cells contain a number of fluorophores. Fluorescence imaging can be used to detect the presence of cancerous cells. It has been found by the authors that abnormalities in the oral lesions increase the intensity ratio of the red to green fluorescence, which can be easily detected by fluorescence imaging. The authors have also found that with fluorescence imaging, the detection rate is $100 \%$ and the specificity is $87.5 \%$, in comparison to white light, whose detection rate and specificity is $87 \%$ and $50 \%$ respectively.

According to the studies carried out by Farah C, et al. [10], the use of VEL scope for detecting epithelial dysplasia by the technology of auto fluorescence imaging provides accuracy of $55 \%$ only.

Again studies by McNamara K, et al. [11] showed that conventional oral examination of mouth is a more valid method of detecting oral precancerous lesion compared to the direct visual fluorescence examination.

The studies also showed that [12] high copy number of mitochondrial DNA in peripheral blood leukocytes has significant association with oral precancerous lesions.

The studies carried out by Baeten J, et al. [13] showed that fluorescence imaging using lectin fluorophore conjugates has more potential to detect oral cancerous lesions compared to the conventional auto fluorescence technology of detection.

The studies by Barroso EM, et al. [14] showed a new method of screening for cancerous cells in the oral mucosa. They used the method of determining water content of the cancerous and the healthy tissues using Raman Spectroscopy. The results showed that the method can be used to detect tumour cells with $99 \%$ sensitivity and $92 \%$ specificity.

There are many more studies that are associated with various other methods of detection and screening of oral cancer.

\section{Evidence based best treatment protocol in relation to the different lesions}

There are many options for the treatment of oral cancers which depend on various factors such as the location and size of the primary lesion, the status of the lymph node, the absence or presence of metastases and the ability of the patient to tolerate. Radiation therapy and surgery are the most common processes that are applied in the treatment of cancerous and precancerous lesions of mouth.

The surgical excision can be performed using $\mathrm{CO}_{2}$ laser or using a scalpel [15]. This is done mainly for the treatment of epithelial dysplasia formed in the oral cavity. The use of laser provides a surgical method that is blood less and less painful compared to the scalpel method.

The lesion should be removed completely in order to prevent any recurrence of the cancer. It has been reported that the rate of recurrence is about $34.4 \%$ in case of premalignant lesions. Again in case of carcinoma or epithelial dysplasia where the excision of the lesion is done with a $3-5 \mathrm{~mm}$ margin, the rate of recurrence is about $18 \%$ [16]. Though the original reason behind the recurrence is unknown, close monitoring of the patient should be done in order to check the recurrence of the cancer.

The removal of the hyperkeratotic lesion is complicated as it may lead to malignant change. According to the studies out of 235 lesions, the 37 lesions changed into malignant.

If there is any difficulty in surgical removal of the lesion due to the size and location of the lesion or due to some medical complications, chemoprevention method is used in order to prevent the progression of precancerous lesions to the cancerous state. The most commonly used chemo-preventive antioxidant supplements in the treatment 
of oral lesions include the retinoid and beta- carotene. But the fact should be taken under consideration that the success rate of antioxidant supplements is not certain. But antioxidants are proved to be helpful if any recurrence occur after the surgical excision. Many studies have shown that treatment with beta carotene only in case of oral leukoplakia has shown significant improvement. The rate of improvement is between $14-71 \%$. Use of beta carotene supplement has not reported any side effects.

It is important to consider that regardless of the method used for the treatment of the cancer, patient faces many problems regarding external feedings, voice change, mucositis, xerostomia, dysphagia and airway protection.

\section{New advances in diagnosing and treating oral soft tissue precancerous and cancerous lesions}

Increased research in the field of oral cancer has resulted in the development of newer technologies for detection and treatment of oral cancers.

The detection techniques that have been developed recently include-

» Fluorescence imaging using lectin fluorophore conjugates

»Auto fluorescence imaging technique using NADH and FAD as fluorophores.

» Another technique includes Light Induced Fluorescence Endoscopy or LIFE to detect early carcinoma.

» Another method of detection include the use of optical spectroscopy in which a fiberoptic probe is used to illuminate as well as collect light from the area of the lesion in order to detect the presence of carcinoma.

» Staining can also be done in order to detect cancerous cells using toluidine blue.

» The studies also suggested the copy number of mitochondrial DNA in peripheral blood leukocytes can also provide an indication of the presence of cancer.

The various newly developed treatment measures include-

» Photodynamic therapy using a photosensitizing agent.

» Other clinical trials include targeting various agents that are associated with cancer development including EGFR inhibitor, COX-2 inhibitor etc.

\section{Conclusion}

With the increase in the use of tobacco and alcohol the risk of oral cancer is increasing. So more studies are required in the field, along with proper control measures and spread of education regarding the fact is needed. Regular test for people with a high risk of developing precancerous and cancerous lesions can make a major effect by the early detection of the disease which leads to more effective and less complicated treatment action. More research is required for more effective treatment considering the low percentage of survival for this complicated disease.

\section{References}

1. Messadi DV (2013) Diagnostic aids for detection of oral precancerous conditions. Int J Oral Sci 5: 59-65.

2. Villa A, Villa C, Abati S (2011) Oral cancer and oral erythroplakia: an update and implication for clinicians. Aust Dent J 56: 253-256.

3. Cox S, Zoellner H (2009) Physiotherapeutic treatment improves oral opening in oral submucous fibrosis. J Oral Pathol Med 38: 220-226.

4. Chaturvedi P (2009) Gutka consumption. Br Dent J 206: 397.

5. Chaturvedi P (2009) Uvular abnormalities in patients with submucous fibrosis. Oral Dis 15: 516.

6. Al-Nasser L, El-Metwally A (2014) Oral lichen planus in Arab countries: a review. J Oral Pathol Med 43: 723-727.

7. Mfoumou E, Li Z, Moustafa A (2013) Current tobacco and waterpipe smoking enhance human cancer invasion and metastasis. Int J Cancer 132: 990-991.

8. Al-Maweri SA, Alaizari NA, Al-Sufyani GA (2014) Oral mucosal lesions and their association with tobacco use and qat chewing among Yemeni dental patients. J Clin Exp Dent 6: e460-e466.

9. Gillenwater A, Papadimitrakopoulou V, Richards-Kortum R (2006) Oral premalignancy: New methods of detection and treatment. Curr Oncol Rep 8: 146-154.

10. Farah C, McIntosh L, Georgiou A, McCullough MJ (2011) Efficacy of tissue autofluorescence imaging (VELScope) in the visualization of oral mucosal lesions. Head Neck 34: 856-862.

11. McNamara K, Martin B, Evans E, Kalmar J (2012) The role of direct visual fluorescent examination (VELscope) in routine screening for potentially malignant oral mucosal lesions. Oral Surg Oral Med Oral Pathol Oral Radiol 114: 636-643.

12. He Y, Gong Y, Gu J, Lee J, Lippman S, et al. (2014) Increased leukocyte mitochondrial DNA copy number is associated with oral premalignant lesions: an epidemiology study. Carcinogenesis 35: 1760-1764.

13. Baeten J, Suresh A, Johnson A, Patel K, Kuriakose M, et al. (2014) Molecular Imaging of Oral Premalignant and Malignant Lesions Using Fluorescently Labeled Lectins. Transl Oncol 7: 213-220.

14. Barroso EM, Smits RW, Bakker Schut TC, ten Hove I, Hardillo JA, et al. (2015) Discrimination between Oral Cancer and Healthy Tissue Based on Water Content Determined by Raman Spectroscopy. Anal Chem 87: 2419-2426.

15. Joris A, Rozenberg, $\mathrm{S}$ (2015) Fractional $\mathrm{CO}_{2}$ laser treatment for atrophic vaginitis in breast cancer patients using aromatase inhibitors. Maturitas 81: 159-160.

16. Al-Bataineh O, Jenne J, Huber P (2012) Clinical and future applications of high intensity focused ultrasound in cancer. Cancer Treat Rev 38: 346-353. 